\title{
SELF-ADJOINTNESS FOR MULTI-POINT DIFFERENTIAL OPERATORS
}

\section{JOHN LOCKER}

\begin{abstract}
In this paper the adjoint operator is derived for a multipoint differential operator in a Hilbert space. This characterization is used to study self-adjointness. In particular, a simple proof is given of some recent results of Neuberger and Zettl.
\end{abstract}

1. Introduction. The adjoint of a multi-point differential operator has been studied by many authors, beginning with Wilder [7] and most recently by Krall [3]. In $\$ 2$ we characterize the adjoint of an $n$th order multi-point differential operator. The development is in the Hilbert space $L^{2}[a, b]$, and it generalizes the setting of Dunford and Schwartz [2, Ch. XIII] for two-point differential operators. The essential feature of this development is the necessity for discontinuities at the interior boundary points, a feature which has been pointed out by Wilder [7] and Loud [4].

In $\S 3$ we characterize self-adjointness for multi-point differential operators, generalizing some classical results of Coddington and Levinson [1]. For the special case of continuity at the interior boundary points, we give an elementary proof of the results of Neuberger [6] and Zettl [8] who have shown that the boundary conditions can not involve the interior boundary points.

2. Multi-point differential operators and their adjoints. For a closed interval $[a, b]$ let $S$ denote the real Hilbert space $L^{2}[a, b]$ with standard inner product $(f, g)$. Let

$$
\tau=\sum_{i=0}^{n} a_{i}(t)\left(\frac{d}{d t}\right)^{i}
$$

be an $n$th order formal differential operator, where we assume that the coefficients $a_{i}(t)$ are real-valued functions belonging to $C^{\infty}[a, b]$ and that $a_{n}(t) \neq 0$ on $[a, b]$. Let $H^{n}[a, b]$ be the linear subspace of $S$ consisting of all functions $f(t)$ in $C^{n-1}[a, b]$ with $f^{(n-1)}$ absolutely continuous on $[a, b]$ and $f^{(n)}$ in $S$.

We are going to study differential operators which are obtained by applying the operator $\tau$ to appropriate linear subspaces of $L^{2}[a, b]$ determined by imposing boundary conditions which involve interior points of $[a, b]$. The following example illustrates some of the problems inherent in determining the adjoint of such an operator. 
EXAMPLE 1. Let $L$ be the differential operator in $L^{2}[-1,1]$ define by

$$
\mathscr{D}(L)=\left\{f \in H^{2}[-1,1] \mid f(0)=f^{\prime}(0)=0\right\}, L f=f^{\prime \prime} .
$$

Since the domain of $L$ is dense in $L^{2}[-1,1]$, the adjoint operator $L^{*}$ exists. Let $f(t)$ be any function in $\mathscr{D}(L)$, and let $g(t)$ be a sufficiently smooth function in $L^{2}[-1,1]$ which possibly has a jump discontinuity at $t=0$. Integrating by parts we obtain

$$
\begin{aligned}
\int_{-1}^{1} f^{\prime \prime} g-\int_{-1}^{1} f g^{\prime \prime}= & f^{\prime}(1) g(1)-f^{\prime}(-1) g(-1)+f(-1) g^{\prime}(-1) \\
& -f(1) g^{\prime}(1)+f(0)\left[g^{\prime}\left(0^{+}\right)-g^{\prime}\left(0^{-}\right)\right] \\
& -f^{\prime}(0)\left[g\left(0^{+}\right)-g\left(0^{-}\right)\right]
\end{aligned}
$$

The last two terms are eliminated by the boundary conditions on $f$, and in the process, the expressions which would have forced continuity on $g$ and $g^{\prime}$ at $t=0$ are also removed. In fact, we see the $g(t)$ belongs to the domain of $L^{*}$ if $g$ is sufficiently smooth on the intervals $[-1,0)$ and $(0,1]$ with $g(1)=g(-1)=g^{\prime}(1)=g^{\prime}(-1)=0$. Note that $g$ and $g^{\prime}$ need not be continuous at $t=0$. Thus, the adjoint operator $L^{*}$ appears to have a different structure than the original differential operator $L$. We will show that this difference is only superficial.

To obtain a theory for multi-point differential operators in which the operator and its adjoint have similar structures, we must extend the concept of such operators by giving up continuity at the interior boundary points of $[a, b]$. Suppose $\pi=\left\{a=x_{0}<x_{1}<\cdots<x_{m}=b\right\}$ is a given partition of $[a, b]$. Let $H^{n}(\pi)$ denote the collection of all functions $f(t)$ in $S$ with the following two properties:

(a) On each subinterval $\left[x_{i-1}, x_{i}\right]$ the function $f(t)$ possesses both right-hand and left-hand limits at the endpoints $x_{i-1}$ and $x_{i}$, respectively. For $i=1, \cdots, m$ let $f_{i}$ be the function defined on $\left[x_{i-1}, x_{i}\right]$ by $f_{i}(t)=$ $f(t)$ for $x_{i-1}<t<x_{i}, f_{i}\left(x_{i-1}\right)=f\left(x_{i-1}^{+}\right)$, and $f_{i}\left(x_{i}\right)=f\left(x_{i}^{-}\right)$. We call the functions $f_{1}, \cdots, f_{m}$ the components of $f$ and denote this by writing $f=\left(f_{1}, \cdots, f_{m}\right)$.

(b) The components $f_{i}$ belong to $H^{n}\left[x_{i-1}, x_{i}\right]$ for $i=1, \cdots, m$. Note that $H^{n}(\pi)$ is a linear subspace of $S$ containing $H^{n}[a, b]$, and we can apply $\tau$ to any function $f(t)$ in $H^{n}(\pi)$ to obtain a new function $\tau f(t)$ in $S$.

We define a multi-point boundary value to be a linear functional $B$ on $H^{n}(\pi)$ of the form

$$
B(f)=\sum_{l=1}^{m} \sum_{j=0}^{n-1}\left[\alpha_{j l} f_{l}^{(j)}\left(x_{l-1}\right)+\beta_{j l} f_{l}^{(j)}\left(x_{l}\right)\right]
$$


for $f=\left(f_{1}, \cdots, f_{m}\right) \in H_{n}(\pi)$, where the $\alpha_{j l}$ and $\beta_{j l}$ are given real numbers. Clearly the space of all such boundary values is a $2 m n$ dimensional linear space.

Suppose we are given a set of $k$ linearly independent multipoint boundary values

$$
B_{i}(f)=\sum_{l=1}^{m} \sum_{j=0}^{n-1}\left[\alpha_{i j l} f_{l}^{(j)}\left(x_{l-1}\right)+\beta_{i j l} f_{l}^{(j)}\left(x_{l}\right)\right], \quad i=1, \cdots, k .
$$

Let $L$ be the linear operator in $S$ defined by

$$
\mathscr{D}(L)=\left\{f \in H^{n}(\pi) \mid B_{i}(f)=0, i=1, \cdots, k\right\}, L f=\tau f .
$$

The operator $L$ is called a multi-point differential operator. Since $\mathscr{D}(L)$ is dense in $S$, we know that $L$ has a well-defined adjoint $L^{*}$. We are going to obtain an explicit formula for $L^{*}$, showing that it is also a multi-point differential operator which is obtained from the formal adjoint $\tau^{*}$ and a set of $2 m n-k$ linearly independent multi-point boundary values.

The principal tool used in studying $L^{*}$ is Green's formula [2, p. 1288]: if $f(t)$ and $g(t)$ are functions in $H^{n}(\pi)$, then

$$
\begin{aligned}
(\tau f, g)-\left(f, \tau^{*} g\right)= & \sum_{l=1}^{m} \sum_{p, q=0}^{n-1}\left[F_{x_{l}}^{p q}(\tau) f_{l}^{(p)}\left(x_{l}\right) g_{l}^{(q)}\left(x_{l}\right)\right. \\
& \left.-F_{x_{l-1}}^{p q}(\tau) f_{l}^{(p)}\left(x_{l-1}\right) g_{l}^{(q)}\left(x_{l-1}\right)\right],
\end{aligned}
$$

where $F_{t}=\left[F_{t}^{p q}(\tau)\right]$ denotes the $n \times n$ boundary matrix for $\tau$ at the point $t \in[a, b]$. Consider the linear system of equations

$$
\sum_{l=1}^{m} \sum_{j=0}^{n-1}\left[\alpha_{i j l} x_{j l}+\beta_{i j l} y_{j l}\right]=0, \quad i=1, \cdots, k .
$$

Since the boundary values $B_{1}, \cdots, B_{k}$ are linearly independent, the system (2) has rank $k$, and hence, the solution space of (2) has dimension $2 m n-k$. Let $\left[x_{i j l}, y_{i j l}\right], i=1, \cdots, 2 m n-k$, be a set of solutions to (2) which form a basis for the solution space.

Next, let $\alpha_{i j l}^{*}$ and $\beta_{i j l}^{*}$ be the constants defined by

$$
\alpha_{i j l}^{*}=-\sum_{p=0}^{n-1} x_{i p l} F_{x_{l-1}}^{p j}(\tau) \quad \text { and } \quad \beta_{i j l}^{*}=\sum_{p=0}^{n-1} y_{i p l} F_{x l}^{p j}(\tau)
$$

for $i=1, \cdots, 2 m n-k ; j=0, \cdots, n-1 ; l=1, \cdots, m$. Let $B_{i}^{*}, i=$ $1, \cdots, 2 m n-k$, be the multi-point boundary values defined by

$$
B_{i}^{*}(f)=\sum_{l=1}^{m} \sum_{j=0}^{n-1}\left[\alpha_{i j l}^{*} f_{l}^{(j)}\left(x_{l-1}\right)+\beta_{i j l}^{*} f_{l}^{(j)}\left(x_{l}\right)\right]
$$

These new boundary values are called adjoint multi-point boundary values. 
THEOREM 1. The adjoint operator $L^{*}$ is the multi-point differential operator defined by

$$
\mathscr{D}\left(L^{*}\right)=\left\{f \in H^{n}(\pi) \mid B_{i}^{*}(f)=0, i=1, \cdots, 2 m n-k\right\}, L^{*} f=\tau^{*} f .
$$

Proof. Let $L_{0}$ be the linear operator in $S$ whose domain consists of all functions $f \in H^{n}(\pi)$ satisfying $B_{i}^{*}(f)=0$ for $i=1, \cdots, 2 m n-k$ with $L_{0} f=\tau^{*} f$. We want to show that $L_{0}=L^{*}$. Fix a function $g \in \mathscr{D}\left(L_{0}\right)$ and set $g^{*}=L_{0} g=\tau^{*} g$. Take any function $f \in \mathscr{D}(L)$. Since the numbers

$$
x_{j l}=f_{l}^{(j)}\left(x_{l-1}\right), \quad y_{j l}=f_{l}^{(j)}\left(x_{l}\right),
$$

form a solution to the linear system (2), there exist constants $c_{1}, \cdots, c_{2 m n-k}$ such that

$$
f_{l}^{(j)}\left(x_{l-1}\right)=\sum_{i=1}^{2 m n-k} c_{i} x_{i j l} \text { and } f_{l}^{(j)}\left(x_{l}\right)=\sum_{i=1}^{2 m n-k} c_{i} y_{i j l} .
$$

Using these equations and Green's formula, we get

$$
\begin{aligned}
(L f, g)-\left(f, g^{*}\right) & =(\tau f, g)-\left(f, \tau^{*} g\right) \\
& =\sum_{i=1}^{2 m n-k} c_{i} B_{i}^{*}(g)=0 .
\end{aligned}
$$

Since this is true for each $f \in \mathscr{D}(L)$, we conclude that $g \in \mathscr{D}\left(L^{*}\right)$ with $L^{*} g=g^{*}=L_{0} g$. Thus, $L_{0} \subseteq L^{*}$.

To complete the proof it is sufficient to show that $\mathscr{D}\left(L^{*}\right) \cong$ $\mathscr{D}\left(L_{0}\right)$. Take any function $g \in \mathscr{D}\left(L^{*}\right)$, and fix an integer $l$ with $1 \leqq l \leqq m$. Let $\bar{g}$ denote the restriction of $g$ to the subinterval $\left[x_{l-1}, x_{l}\right]$. If $\bar{f}$ is any function in $H^{n}\left[x_{l-1}, x_{l}\right]$ having its support in the open interval $\left(x_{l-1}, x_{l}\right)$, then we can extend $\bar{f}$ to a function $f$ defined on $[a, b]$ by making it zero outside of $\left[x_{l-1}, x_{l}\right]$; the extension $f$ belongs to $\mathscr{D}(L)$, and hence,

$$
0=(L f, g)-\left(f, L^{*} g\right)=\int_{x_{l-1}}^{x_{l}}(\tau \bar{f}) \bar{g}-\int_{x_{l-1}}^{x_{l}} \bar{f}\left(L^{*} g\right) .
$$

By Theorem 10 of $[2$, p. 1294] we conclude that $\bar{g}$ is equal a.e. to a function in $H^{n}\left[x_{l-1}, x_{l}\right]$ and that $L^{*} g=\tau^{*} \bar{g}$ a.e. on $\left[x_{l-1}, x_{l}\right]$. Thus, $g \in H^{n}(\pi)$ and $L^{*} g=\tau^{*} g$. Next, fix an integer $i$ with $1 \leqq i \leqq 2 m n-k$, and choose a function $\sigma=\left(\sigma_{1}, \cdots, \sigma_{m}\right) \in H^{n}(\pi)$ such that $\sigma_{l}^{(j)}\left(x_{l-1}\right)=$ $x_{i j l}$ and $\sigma_{l}^{(j)}\left(x_{l}\right)=y_{i j l}$. Clearly $\sigma \in \mathscr{D}(L)$, and from Green's formula

$$
0=(L \sigma, g)-\left(\sigma, L^{*} g\right)=(\tau \sigma, g)-\left(\sigma, \tau^{*} g\right)=B_{i}^{*}(g),
$$

so $g \in \mathscr{D}\left(L_{0}\right)$. This completes the proof.

For calculating adjoints and for studying self-adjointness, it is 
convenient to introduce the following matrices. Let $M_{l}$ and $N_{l}$ be the $k \times n$ matrices defined by

$$
M_{l}=\left[\alpha_{i j l}\right] \text { and } N_{l}=\left[\beta_{i j l}\right],
$$

let $P_{l}$ and $Q_{l}$ be the $(2 m n-k) \times n$ matrices defined by

$$
P_{l}=\left[\alpha_{i j l}^{*}\right] \text { and } Q_{l}=\left[\beta_{i j l}^{*}\right] \text {, }
$$

and let $X_{l}$ and $Y_{l}$ be the $(2 m n-k) \times n$ matrices defined by

$$
X_{l}=\left[x_{i j l}\right] \text { and } Y_{l}=\left[y_{i j l}\right]
$$

for $l=1, \cdots, m$. From (3) we have

$$
P_{l}=-X_{l} F_{x_{l-1}} \text { and } Q_{l}=Y_{l} F_{x_{l}}
$$

for $l=1, \cdots, m$. Finally, let $X$ and $X^{*}$ be the $(2 m n-k) \times 2 m n$ matrices

$$
X=\left[X_{1} Y_{1} \cdots X_{m} Y_{m}\right] \text { and } X^{*}=\left[P_{1} Q_{1} \cdots P_{m} Q_{m}\right],
$$

and let $F$ be the $2 m n \times 2 m n$ matrix

$$
F=\left[\begin{array}{cccc}
-F_{x_{0}} & & & 0 \\
& F_{x_{1}} & & \\
& \ddots & & \\
& & & -F_{x_{m-1}} \\
& & & \\
0 & & & F_{x_{m}}
\end{array}\right] .
$$

From (5) we have

$$
X^{*}=X F
$$

REMARK. We know that $X$ has rank $2 m n-k$ because system (2) has rank $k$, and that $F$ is nonsingular because the boundary matrix $F_{t}$ is nonsingular at each point $t \in[a, b]$, and hence, $X^{*}$ must have rank $2 m n-k$. Since the rows of $X^{*}$ are the coefficients of the adjoint multi-point boundary values, it follows that $B_{1}^{*}, \cdots, B_{2 m n-k}^{*}$ are linearly independent.

We conclude this section by giving two examples. In the first we reexamine Example 1, while in the latter we look at an example due to Loud [4, p. 316].

ExAmple 2. The first example can be reformulated as follows: let $S=L^{2}[-1,1]$, let $\tau f=f^{\prime \prime}$, let $\pi=\{-1,0,1\}$, and let 


$$
\begin{aligned}
& B_{1}(f)=f\left(0^{-}\right)-f\left(0^{+}\right)=f_{1}(0)-f_{2}(0), \\
& B_{2}(f)=f^{\prime}\left(0^{-}\right)-f^{\prime}\left(0^{+}\right)=f_{1}^{\prime}(0)-f_{2}^{\prime}(0), \\
& B_{3}(f)=f\left(0^{-}\right)=f_{1}(0), \\
& B_{4}(f)=f^{\prime}\left(0^{-}\right)=f_{1}^{\prime}(0),
\end{aligned}
$$

so the operator $L$ is given by

$$
\mathscr{D}(L)=\left\{f \in H^{2}(\pi) \mid B_{i}(f)=0, i=1, \cdots, 4\right\}, L f=f^{\prime \prime} .
$$

For the adjoint operator we have $\tau^{*} f=f^{\prime \prime}$ and

$$
F_{t}=\left[\begin{array}{rr}
0 & -1 \\
1 & 0
\end{array}\right] \text { for all } t \in[-1,1] \text {. }
$$

The linear system (2) is given by

$$
\begin{aligned}
& 0 x_{01}+0 x_{11}+1 y_{01}+0 y_{11}-1 x_{02}+0 x_{12}+0 y_{02}+0 y_{12}=0, \\
& 0 x_{01}+0 x_{11}+0 y_{01}+1 y_{11}+0 x_{02}-1 x_{12}+0 y_{02}+0 y_{12}=0, \\
& 0 x_{01}+0 x_{11}+1 y_{01}+0 y_{11}+0 x_{02}+0 x_{12}+0 y_{02}+0 y_{12}=0, \\
& 0 x_{01}+0 x_{11}+0 y_{01}+1 y_{11}+0 x_{02}+0 x_{12}+0 y_{02}+0 y_{12}=0,
\end{aligned}
$$

which has as its general solution

$$
x_{01}[10000000]+x_{11}[01000000]+y_{02}[00000010]+y_{12}[00000001] \text {. }
$$

Thus,

$$
X=\left[\begin{array}{llllllll}
1 & 0 & 0 & 0 & 0 & 0 & 0 & 0 \\
0 & 1 & 0 & 0 & 0 & 0 & 0 & 0 \\
0 & 0 & 0 & 0 & 0 & 0 & 1 & 0 \\
0 & 0 & 0 & 0 & 0 & 0 & 0 & 1
\end{array}\right] \text { and } X^{*}=X F=\left[\begin{array}{rrrrrrrr}
0 & 1 & 0 & 0 & 0 & 0 & 0 & 0 \\
-1 & 0 & 0 & 0 & 0 & 0 & 0 & 0 \\
0 & 0 & 0 & 0 & 0 & 0 & 0 & -1 \\
0 & 0 & 0 & 0 & 0 & 0 & 1 & 0
\end{array}\right]
$$

It follows that adjoint multi-point boundary values are given by

$$
\begin{aligned}
& B_{1}^{*}(f)=f^{\prime}(-1)=f_{1}^{\prime}(-1), \\
& B_{2}^{*}(f)=-f(-1)=-f_{1}(-1), \\
& B_{3}^{*}(f)=-f^{\prime}(1)=-f_{2}^{\prime}(1), \\
& B_{4}^{*}(f)=f(1)=f_{2}(1),
\end{aligned}
$$

and hence, the adjoint operator $L^{*}$ is given by

$$
\mathscr{D}\left(L^{*}\right)=\left\{f \in H^{2}(\pi) \mid f(-1)=f^{\prime}(-1)=f(1)=f^{\prime}(1)=0\right\}, L^{*} f=f^{\prime \prime} .
$$

ExAMPLE 3. Let $S=L^{2}[-1,1]$, let $\tau f=f^{\prime \prime}$, let $\pi=\{-1,0,1\}$, and let 


$$
\begin{aligned}
& B_{1}(f)=f(-1)=f_{1}(-1), \\
& B_{2}(f)=f(1)=f_{2}(1), \\
& B_{3}(f)=f\left(0^{-}\right)-f\left(0^{+}\right)=f_{1}(0)-f_{2}(0), \\
& B_{4}(f)=f\left(0^{-}\right)-f^{\prime}\left(0^{-}\right)+f^{\prime}\left(0^{+}\right)=f_{1}(0)-f_{1}^{\prime}(0)+f_{2}^{\prime}(0) .
\end{aligned}
$$

For the operator $L$ determined by these conditions, we find that

$$
X=\left[\begin{array}{llllllll}
0 & 1 & 0 & 0 & 0 & 0 & 0 & 0 \\
0 & 0 & 1 & 1 & 1 & 0 & 0 & 0 \\
0 & 0 & 0 & 1 & 0 & 1 & 0 & 0 \\
0 & 0 & 0 & 0 & 0 & 0 & 0 & 1
\end{array}\right], \quad X^{*}=\left[\begin{array}{rrrrrrrr}
-1 & 0 & 0 & 0 & 0 & 0 & 0 & 0 \\
0 & 0 & 1 & -1 & 0 & 1 & 0 & 0 \\
0 & 0 & 1 & 0 & -1 & 0 & 0 & 0 \\
0 & 0 & 0 & 0 & 0 & 0 & 1 & 0
\end{array}\right],
$$

and hence, adjoint multi-point boundary values are given by

$$
\begin{aligned}
& B_{1}^{*}(f)=-f(-1)=-f_{1}(-1), \\
& B_{2}^{*}(f)=f\left(0^{-}\right)-f^{\prime}\left(0^{-}\right)+f^{\prime}\left(0^{+}\right)=f_{1}(0)-f_{1}^{\prime}(0)+f_{2}^{\prime}(0), \\
& B_{3}^{*}(f)=f\left(0^{-}\right)-f\left(0^{+}\right)=f_{1}(0)-f_{2}(0), \\
& B_{4}^{*}(f)=f(1)=f_{2}(1) .
\end{aligned}
$$

Thus, $L=L^{*}$.

3. Self-adjointness. Since the rows of the matrix $X$ are solutions of the linear system (2), we have

$$
\sum_{l=1}^{m}\left[M_{l} X_{l}^{t}+N_{l} Y_{l}^{t}\right]=0,
$$

and hence, substituting (5) into this equation we get

$$
\sum_{l=1}^{m}\left[M_{l}\left(-F_{x_{l-1}}^{-1}\right)^{t} P_{l}^{t}+N_{l}\left(F_{x_{l}}^{-1}\right)^{t} Q_{l}^{t}\right]=0 .
$$

We are going to use this equation to characterize the self-adjoint multi-point differential operators. In case $m=1$ equation (7) reduces to equation (3.3) of Coddington and Levinson [1, p. 289].

Theorem 2. Let $\tau=\tau^{*}$ and let $k=m n$. The multi-point differential operator $L$ is self-adjoint if $f$

$$
\sum_{l=1}^{m}\left[M_{l}\left(-F_{x_{l-1}}^{-1}\right)^{t} M_{l}^{t}+N_{l}\left(F_{x_{l}}^{-1}\right)^{t} N_{l}^{t}\right]=0 .
$$

Proof. First, assume $L=L^{*}$. Since the boundary values $\mathrm{B}_{1}, \cdots, B_{m n}$ are equivalent to the boundary values $B_{1}^{*}, \cdots, B_{m n}^{*}$, there exists an $m n \times m n$ nonsingular matrix $A_{1}$ such that

$$
\left[P_{1} Q_{1} \cdots P_{m} Q_{m}\right]=A_{1}\left[M_{1} N_{1} \cdots M_{m} N_{m}\right],
$$


and hence, $P_{l}=A_{1} M_{l}$ and $Q_{l}=A_{1} N_{l}$ for $l=1, \cdots, m$. Substituting these results into equation (7) yields equation (8).

Conversely, assume equation (8) is true. This implies that the rows of the $m n \times 2 m n$ matrix $\left[M_{1} N_{1} \cdots M_{m} N_{m}\right] F^{-1}$ are solutions to the linear system (2). Because these rows are linearly independent, there exists an $m n \times m n$ nonsingular matrix $A_{2}$ such that

$$
\left[X_{1} Y_{1} \cdots X_{m} Y_{m}\right]=X=A_{2}\left[M_{1} N_{1} \cdots M_{m} N_{m}\right] F^{-1} .
$$

Therefore,

$$
\left[P_{1} Q_{1} \cdots P_{m} Q_{m}\right]=X F=A_{2}\left[M_{1} N_{1} \cdots M_{m} N_{m}\right] .
$$

This implies that the boundary values $B_{1}, \cdots, B_{m n}$ and $B_{1}^{*}, \cdots, B_{m n}^{*}$ are equivalent. Thus, $L=L^{*}$ and the proof is complete.

REMARK 1 . In case $m=1$ this theorem reduces to Theorem 3.2 of Coddington and Levinson [1, p. 291].

REMARK 2. Loud [4, Theorem 1, p. 309] has also obtained a characterization of self-adjoint multi-point differential operators. In applying his results one must know a fundamental matrix for the homogeneous problem and know that a Green's function exists. These restrictions do not apply to Theorem 2, and consequently, it may be easier to use in practical problems.

We conclude this paper by examining self-adjointness in the special case of continuity at the interior boundary points. Suppose $L$ is a self-adjoint multi-point differential operator determined by $\tau$ and multi-point boundary values $B_{1}, \cdots, B_{m n}$, and assume that the last $(m-1) n$ of these boundary values specify continuity for the functions and their derivatives at the interior boundary points:

$$
f_{l}^{(j)}\left(x_{l}^{-}\right)-f_{l}^{(j)}\left(x_{l}^{+}\right)=f_{l}^{(j)}\left(x_{l}\right)-f_{l+1}^{(j)}\left(x_{l}\right)=0,
$$

for $l=1, \cdots, m-1, j=0 \cdots, n-1$. Note that the boundary values $B_{1}, \cdots, B_{n}$ can be rewritten in the form

$$
B_{i}(f)=\sum_{l=0}^{m} \sum_{j=0}^{n-1} \gamma_{i j l} f_{l}^{(j)}\left(x_{l}\right), \quad i=1, \cdots, n,
$$

and $L$ can be represented by

$$
\mathscr{D}(L)=\left\{f \in H^{n}[a, b] \mid B^{i}(f)=0, i=1, \cdots, n\right\}, L f=\tau f .
$$

We assert that the coefficients $\gamma_{i j l}$ in (10) which correspond to interior boundary points are all 0 .

Fix integers $i, j, l$ with $1 \leqq i \leqq n, 0 \leqq j \leqq n-1,1 \leqq l \leqq m-1$, and suppose $\gamma_{i j l} \neq 0$. Let $L_{0}$ be the multi-point differential operator 
determined by $\tau$ and the boundary conditions (9) together with the additional boundary condition $B_{i}(f)=0$. Clearly $L \cong L_{0}$, and hence, $L_{0}^{*} \leqq L^{*}=L$. Also, since $L_{0}$ is given by $(m-1) n+1$ linearly independent boundary conditions, the adjoint $L_{0}^{*}$ is determined by precisely $2 m n-(m-1) n-1=m n+n-1$ linearly independent boundary conditions.

Choose a function $f \in H^{n}[a, b]$ with

$$
\begin{aligned}
{\left[f(a) f^{\prime}(a) \cdots f^{(n-1}(a)\right] } & =-\left[\begin{array}{llll}
1 & 0 & \cdots & 0
\end{array}\right] F_{a}^{-1}, \\
{\left[f(b) f^{\prime}(b) \cdots f^{(n-1}(b)\right] } & =\left[\begin{array}{llll}
0 & 0 & \cdots & 0
\end{array}\right] \\
f^{(j)}\left(x_{l}\right) & =-\left(\gamma_{i j l}\right)^{-1} \sum_{p=0}^{n-1} \gamma_{i p 0} f^{(p)}(a),
\end{aligned}
$$

and with all other values for $f, f^{\prime}, \cdots, f^{(n-1)}$ equal to 0 at the interior boundary points. Note that $f \in \mathscr{D}\left(L_{0}\right)$. Take any function $g \in \mathscr{D}\left(L_{0}^{*}\right)$. Since $\mathscr{D}\left(L_{0}^{*}\right) \subseteq \mathscr{D}(L)$, it follows that $g$ and its derivatives are continuous at the interior boundary points, and hence, using Green's formula we get

$$
\begin{aligned}
\mathbf{0}= & \left(L_{0} f, g\right)-\left(f, L_{0}^{*} g\right) \\
= & {\left[f(b) f^{\prime}(b) \cdots f^{(n-1)}(b)\right] F_{b}\left[g(b) g^{\prime}(b) \cdots g^{(n-1)}(b)\right]^{t} } \\
& -\left[f(a) f^{\prime}(a) \cdots f^{(n-1)}(a)\right] F_{a}\left[g(a) g^{\prime}(a) \cdots g^{(n-1)}(a)\right]^{t} \\
= & g(a) .
\end{aligned}
$$

Using this same argument we can show that $g^{(p)}(a)=0$ and $g^{(p)}(b)=$ 0 for $p=0,1, \cdots, n-1$ for all functions $g \in \mathscr{D}\left(L_{0}^{*}\right)$. These $2 n$ boundary conditions together with the $(m-1) n$ boundary conditions (9) account for a total of $2 n+(m-1) n=m n+n$ linearly independent boundary conditions satisfied by the functions $g \in \mathscr{D}\left(L_{0}^{*}\right)$. This contradicts the earlier statement that $\mathscr{D}\left(L_{0}^{*}\right)$ is determined by $m n+n-1$ linearly independent boundary conditions. Therefore, we must have $\gamma_{i j l}=0$, and we have established the following theorem.

THEOREM 3. Let $L$ be a self-adjoint multi-point differential operator determined by $\tau$ and multi-point boundary values $B_{1}, \cdots, B_{m n}$. If the last $(m-1) n$ of these boundary values specify continuity for the functions and their derivatives at the interior boundary points as in (9), then in the representations for the boundary values $B_{1}, \cdots, B_{n}$ given in (10) the coefficients $\gamma_{i j l}$ which correspond to interior boundary points are all 0.

REMark. This theorem has been established by Neuberger [6] and Zettl [8]. Our proof seems simpler and more natural. It is a 
consequence of Green's formula, which is the basic principle used in studying self-adjointness.

\section{REFERENCES}

1. E. A. Coddington and N. Levinson, Theory of Ordinary Differential Equations, McGraw-Hill, New York, 1955.

2. N. Dunford and J. T. Schwartz, Linear Operators, I and II, Interscience, New York, 1958 and 1963.

3. A. M. Krall, Boundary value problems with interior point boundary conditions, Pacific J. Math., 29 (1969), 161-166.

4. W. S. Loud, Self-adjoint multi-point boundary value problems, Pacific J. Math., 24 (1968), 303-317.

5. R. Mansfield, Differential systems involving k-point boundary conditions, Contributions to the Calculus of Variations 1938-1914, Chicago, 1942, 413-452.

6. J. W. Neuberger, The lack of self-adjointness in three point boundary value problems, Pacific J. Math., 18 (1966), 165-168.

7. C. E. Wilder, Problems in the theory of linear differential equations with auxiliary conditions at more than two points, Trans. Amer. Math. Soc., 19 (1918), 157-166.

8. A. Zettl, The lack of self-adjointness in three point boundary value problems, Proc. Amer. Math. Soc., 17 (1966), 368-371.

9. - Adjoint and self-adjoint boundary value problems with interface conditions, SIAM J. Appl. Math., 16 (1968), 851-859.

Received February 24, 1972.

Colorado State University 